\title{
ORIGINAL
}

\section{The plasma level changes of VEGF and soluble VEGF receptor-1 are associated with high-altitude pulmonary edema}

\author{
Shukun Zhang ${ }^{1,2}$, Juanli Liu³ ${ }^{3}$ Dongmei Jiang ${ }^{4}$, Tana Wuren ${ }^{5}$, Siqing $\mathrm{Ma}^{6}$, Yansheng $\mathrm{Du}^{7}$, Xin $\mathrm{Yi}^{7}$, and Shizheng $\mathrm{Wu}^{8}$ \\ ${ }^{1}$ Department of Pathology, Weihai Municipal Hospital, Weihai, Shandong, China, ${ }^{2}$ Department of Pathology, Qinghai Provincial People's \\ Hospital, Xining, Qinghai, China, ${ }^{3}$ Department of Internal Medicine, Qinghai Provincial People's Hospital, Xining, Qinghai, China, ${ }^{4}$ Institute for \\ Neurological diseases, Qinghai Provincial People's Hospital, Xining, Qinghai, China, ${ }^{5}$ Research Centre for High Altitude Medicine, Qinghai \\ University Medical College, Xining, Qinghai, China, ${ }^{6}$ Department of Intensive Care Unit, Qinghai Provincial People's Hospital, Xining, \\ Qinghai, China, ${ }^{7}$ Department of Neurology, Indiana University School of Medicine, Indianapolis, IN, ${ }^{8}$ Department of Neurology, Qinghai \\ Provincial People's Hospital, Xining, Qinghai, China
}

\begin{abstract}
Hypoxia-induced plasma levels of VEGF and sFlt-1 are responsible for increased vascular permeability occurred in both brain and pulmonary edema. Currently, it remains unclear the exact roles of VEGF and sFlt-1 in High Altitude Pulmonary Edema (HAPE) pathogenesis. In this study, plasma levels of VEGF and sFlt-1 from 10 HAPE and 10 non-HAPE subjects were measured and compared. The results showed that plasma levels of both VEGF and sFlt-1 in HAPE patients were significantly increased as compared to the non-HAPE group. Interestingly, increased plasma levels of these two protein factors were markedly reduced after treatments. As compared to VEGF, sFlt-1 was much more affected by hypoxia and treatments, suggesting this factor was a key factor contributed to HAPE pathogenesis. Importantly, the ratio of sFlt- 1 and VEGF in group of either non - HAPE or HAPE after recovery was significantly lower than the ratio in HAPE patients prior to treatments. Our findings suggested that sFlt-1 was a key factor that involved in HAPE pathogenesis and the sFlt-1/VEGF ratio could be used as a sensitive diagnostic marker for HAPE. J. Med. Invest. 65 : 64-68, February, 2018
\end{abstract}

Keywords : Vascular endothelial growth factor, Soluble VEGF receptor, High altitude pulmonary edema

\section{INTRODUCTION}

Acute mountain sickness (AMS) is a hypoxia-induced symptom at high altitude and can progress to high altitude pulmonary edema (HAPE) or high altitude cerebral edema (HACE) (1). HAPE is a lifethreatening non-cardiogenic pulmonary edema that occurs in many mountaineers at high altitudes with the high mortality rate (2) and remains to be the major cause of death in the absence of adequate emergency treatment. In general, HAPE is caused by hypoxiainduced pulmonary artery pressure that results in interstitial and alveolar pulmonary edema, as well as impaired oxygenation (3). According to the Lake Louise Consensus, in the context of a recent ascent, patients with HAPE will have some combination of the following symptoms and signs, including dyspnea at rest, cough, weakness or decreased exercise performance, chest tightness, central cyanosis, tachypnea, and tachycardia. In practice, the diagnosis is usually clinically apparent. The treatment of HAPE includes descent, rest, and administration of oxygen or Dexamethasone. However, treatments for this disease vary considerably because the underlying pathophysiologic mechanisms remain unclear. The most possible and crucial pathological mechanism for HAPE is hypoxia-induced capillary permeability (4). Recently, much work has focused on the role of vascular endothelial growth factor

Received for publication February 20, 2017 ; accepted November 22, 2017.

Address correspondence and reprint requests to Xin Yi, $\mathrm{PhD}$, Department of Neurology, School of Medicine, Indiana University, $975 \mathrm{~W}$ Walnut Street, Indianapolis, IN 46202, Fax : +01(317) 274-3587, and Shizheng $\mathrm{Wu}, \mathrm{MD}, \mathrm{PhD}$, Department of Neurology, Qinghai Provincial People's Hospital, 2 Gonghe Rd, Chengdong, Xining, Qinghai, China, 810007, China and Fax : +86-0971-8066302.
(VEGF) that is mainly expressed in lung and brain choroid plexus and demonstrated it is a potent vascular permeability factor under hypoxia(5). However, it remains to be determined whether circulating VEGF in blood is a key factor that contributes to the pathogenesis of HAPE since VEGF levels are not consistently increased in AMS patients $(6,7)$. Soluble fms-like tyrosine kinase-1 (sFlt-1) is a splice variant of VEGF receptor which is produced by a variety of tissues (8) and is able to bind VEGF to reduce its free circulating levels and bioactivity (9). Interestingly, Flt-1 levels are also dramatically stimulated in most of cases of hypoxia (10). It has been reported that in addition to bind VEGF, Flt-1 also antagonizes and reduces bioactivities of other pro-vascular permeability factors such as PIGF (11), semaphoring 3A (Sema3A) (12) and Galectin-1 (Gal-1) (13). In HAPE, however, the role of sFlt-1 remains unknown. Currently, two studies have showed sFlt-1 plays the insignificant role in AMS $(7,14)$.

In this study, we have studied and demonstrated the potential roles of plasma VEGF and sFlt-1 in HAPE development by comparing plasma levels of VEGF and sFlt-1 among cohorts of amateur mountaineers with or without HAPE in Qinghai province, China.

\section{METHODS}

Research subjects

Selected healthy subjects were selected by using following predetermined criteria : at age range 20-45ys, no significant medical conditions including diabetes, hypertension, cardiovascular disease and chronic pulmonary disease, no recent exposure to high altitude, no history of HAPE or HACE, and not involved in professional climbing/mountaineering training. The human research 
ethical committee from Qinghai Provincial Hospital approved the study protocol and all subjects signed written informed consent form.

Research subjects participated in three mountaineering activities in Golmud (2800 m) and Yuzhu Peak $(5050 \mathrm{~m})$ between May 2013 and Oct 2013.The Lake Louise Score evaluation for acute mountain sickness (AMS) and clinical assessments were performed on day $0(2800 \mathrm{~m})$, day $3(4500 \mathrm{~m})$, and day $5(5050 \mathrm{~m})$. The Lake Louis Consensus definition of HAPE was defined as in the setting of recentaltitude gain with the presence of the at least two of symptoms : dyspnoea at rest, weakness or decreased exercise performance, cough, chest tightness or congestion, and the presence of at least two of signs : tachypnoea, tachycardia, crackles, or wheezing in at least one lung field, and central cyanosis (15).

\section{Evaluation of HAPE severity}

According to Lake Louise Criteria, the diagnosis of HAPE was made by at least two of the following symptoms : shortness of breath at rest, cough, weakness and decreased exercise performance, chest tightness or congestion ; AND at least two of the following signs on physical exam : fast heart rate, fast breathing, crackles or wheezing heard in the lungs, or low oxygen measured by a pulse oximeter. Chest X-ray in HAPE patient confirms fluid in the lungs. After hospitalization, patients were treated immediatedly with bed rest, oxygen, Dexamethasone. Demographic and clinical features immediately recorded included age, sex, self-reported previous history of HAPE, rate of ascent, altitude at onset of symptoms, physical examination findings, and vital signs including pulse oximetry were recorded. HAPE patients were classified into three groups : mild, moderate, and severe (16). Among 20 subjects who were born and resided at low altitude, 10 did not have any symptoms during all activities, and another 10 developed HAPE. All HAPE patients were transferred to hospital within 2-3 hours. The diagnosis of HAPE was confirmed and patients were recovered with disappearance of symptoms and signs during 3 days of treatments with bed rest and supplemental oxygen, and chest radiographic infiltrates consistent with pulmonary edema.

\section{Blood samples}

Venous blood was drawn from the patients with HAPE at the time of admission before treatment and discharge from the hospital. Meanwhile, the blood samples were also drowned from nonHAPE subjects right after they arrived at the low $(2800 \mathrm{~m})$ and the high altitude camp $(5050 \mathrm{~m})$; all the blood samples were transported with ice, centrifuged immediately after receiving at 3000 $\mathrm{rpm}$ for $15 \mathrm{~min}$, and stored at $-80^{\circ} \mathrm{C}$ until assays.

\section{ELISA assay}

Plasma levels of sFlt-1 and VEGF were determined in duplicate using Quantikine ELISA kits for human VEGF and Flt-1 (R\&D Systems, Minneapolis, USA) according to the manufacture guidance. For plasma VEGF or sFlt-1 quantification, $100 \mu \mathrm{l}$ of standards and undiluted plasma were added and incubated for $1 \mathrm{~h}$ at room temperature in the 96 -well plate coated with monoclonal VEGF or sFlt- 1 capture antibody.

\section{Statistics}

Paired Student's t-test was used to analyze the data obtained from HAPE patients before and after treatment. Whitney U test was used to analyze data obtained from HAPE patients and non-HAPE subjects. Correlations between VEGF and/or sFlt-1 and HAPE severity were also determined. Data are expressed as mean \pm S.E. Significance was set at $\mathrm{p}<0.05$ for all statistical analyses.

\section{RESULTS}

The anthropometric plasma levels of VEGF and sFlt-1 in all subjects were determined and presented in Table 1 . After treatment, monitoring indexes, including $\mathrm{HR}, \mathrm{SpO}_{2}$, and body temperature were significantly improved comparing to pre-treatment. Plasma levels of both VEGF and sFlt-1 in HAPE patients were dramatically increased as compared to non-HAPE at the same altitude $(5050 \mathrm{~m}$ ) (VEGF : $1117.74 \pm 46.98 \mathrm{pg} / \mathrm{ml}$ vs. $432.86 \pm 103.38$ $\mathrm{pg} / \mathrm{ml}, \mathrm{p}<0.01 ; \mathrm{sFlt}-1: 389.73 \pm 76.89 \mathrm{pg} / \mathrm{ml}$ vs. $15.42 \pm 5.39 \mathrm{pg} /$ $\mathrm{ml}, \mathrm{p}<0.01$ ) (Figure 1, A, B). Interestingly, there was a 25-fold increase in sFlt-1 levels as compared to a 2.6-fold increase in VEGF levels. As expected, in HAPE patients, elevated plasma levels of VEGF and sFlt- 1 in patients were significantly reduced after patients were recovered in the hospital (VEGF : $1117.74 \pm 47.01$ vs $252 \pm 24.56, \mathrm{p}<0.01$; sFlt $-1: 389.73 \pm 24.33$ vs $22.34 \pm 6.96, \mathrm{p}<0.01$ ) (Figure 2, A, B). Again, sFlt-1 levels were decreased much more than VEGF levels after recovery (4.4 fold vs. 17.4 fold). Clearly, sFlt- 1 changes were much bigger during hypoxia and treatments. Additionally, ratios of sFlt-1 and VEGF were compared between the non-HAPE group and the before treatment group in HAPE patients $(0.08 \pm 0.03$ vs $0.35 \pm 0.02, \mathrm{p}<0.01)$ or the after treatment group $(0.09 \pm 0.03$ vs. $0.25 \pm 0.04, \mathrm{p}<0.05)$. We found the sFlt- 1 alteration without considering VEGF effects might be more meaningful for patient assessments (Figure 3, A, B).

Table 1 : The anthropometric data analysis of subjects

\begin{tabular}{cccc}
\hline Characteristic & $\begin{array}{c}\text { Non-HAPE } \\
\text { Conrols } \\
(\mathrm{n}=10)\end{array}$ & \multicolumn{2}{c}{$\begin{array}{c}\text { HAPE Patients } \\
(\mathrm{n}=10)\end{array}$} \\
\hline & & $\begin{array}{c}\text { Before } \\
\text { treatment }\end{array}$ & $\begin{array}{c}\text { After } \\
\text { treatment }\end{array}$ \\
Age, yrs & $29.4 \pm 10.04$ & \multicolumn{2}{c}{$30.08 \pm 5.00$} \\
Height, cm & $175.22 \pm 3.17$ & $172.00 \pm 4.43$ \\
Weight, $\mathrm{Kg}$ & $72.00 \pm 7.15$ & \multicolumn{2}{c}{$68.70 \pm 7.42$} \\
$\mathrm{BMI}, \mathrm{Kg} / \mathrm{m} 2$ & $22.85 \pm 3.38$ & $22.63 \pm 3.22$ \\
$\mathrm{SBP}, \mathrm{mmHg}$ & $119.92 \pm 18.32$ & $118.91 \pm 7.54$ & $120.86 \pm 4.62$ \\
$\mathrm{DBP}, \mathrm{mmHg}$ & $83.28 \pm 11.56$ & $77.10 \pm 3.78$ & $72.74 \pm 1.82$ \\
HR, min-1 & $78.25 \pm 10.56$ & $113.06 \pm 18.90$ & $82.53 \pm 12.83 \star$ \\
SpO $2, \%$ & $91.05 \pm 1.41$ & $65.98 \pm 3.62$ & $89.72 \pm 1.94 \star$ \\
Temperature & & \multicolumn{2}{c}{} \\
(Celsius & $36.77 \pm 0.09$ & $36.53 \pm 0.10$ & $37.3 \pm 0.08^{\star}$ \\
degree) & & & \\
\hline
\end{tabular}

$\star$ Significant between before treatment and after treatment group.

\section{DISCUSSION}

It was well accepted that VEGF induced pulmonary hypertension and vascular permeability after its binding to endothelial cells (17). VEGF is a powerful mediator of capillary permeability by disrupting basement membrane ligands, thus increasing capillary permeability and leading to plasma leak from capillaries (18). Previous studies have evaluated the circulating VEGF in the pathogenesis of AMS, however, the function of VEGF in HAPE remain inconclusive ; As an antagonist, sFlt-1 was able to sequester circulating VEGF to inhibit its binding to the endothelium-bound receptors to stimulate VEGF-induced vascular permeability . However, it remained unclear why both factors were sometimes increased together in hypoxia and whether/how these two factors could be 
$\mathrm{A}$

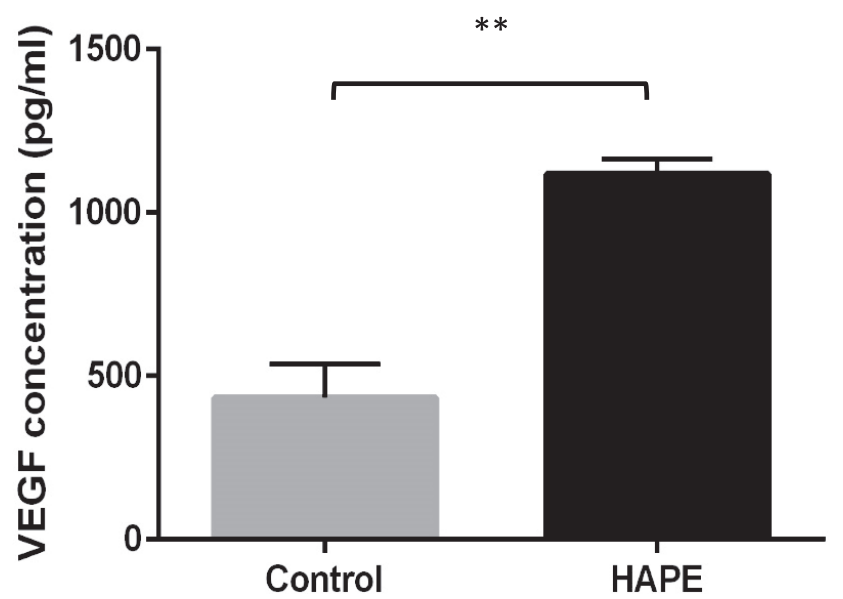

B

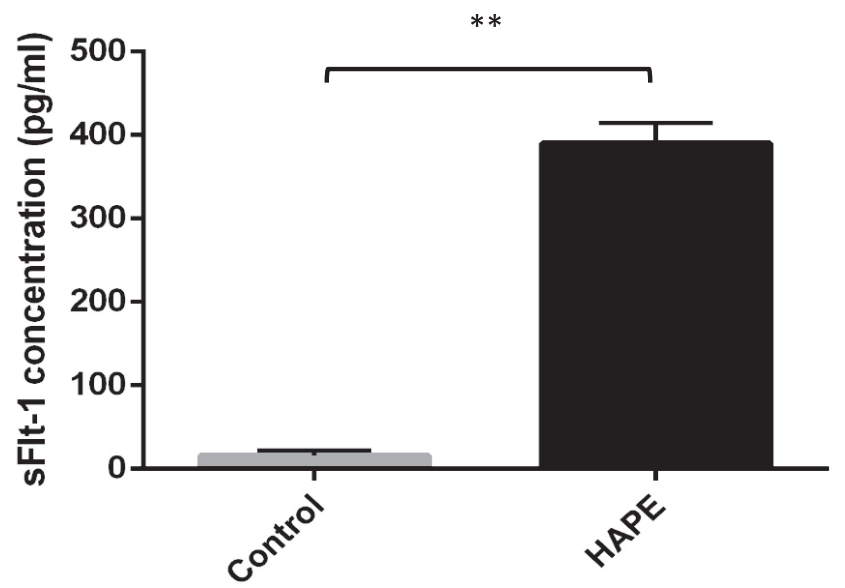

Figure 1. Plasma levels of VEGF and sFlt-1 in HAPE $(n=10)$ and control $(\mathrm{n}=10)$ subjects at Yuzhu peak camp $(5050 \mathrm{~m})$. A, the mean plasma level of VEGF in HAPE patients was significantly increased as compared with control subjects. B, the plasma level of sFlt-1 was significantly increased in subjects who developed HAPE as compared to control individuals. ${ }^{* *} \mathrm{p}<0.01$.

used to assess the hypoxia condition in HAPE. Importantly, several studies suggested that these two factors were not consistently induced in AMS. Most studies showed that circulating VEGF was significantly increased in AMS or HAPE patients at high altitude (7, 19). However, at least in one study, plasma VEGF level was little changed between AMS + and AMS- subjects at high altitude (14). In sFlt-1 studies, Schommer showed sFlt-1 was increased but not significantly between AMS+ and AMS-(14). Another study even demonstrated that AMS subjects had lower sFlt-1 levels at both low and high altitude compared to control subjects (7). In addition, the level of VEGF in this study was much higher than those in previous research. We believed these discrepancies might be attributable to different sample collecting time. Among these studies and our study, we clearly showed that plasma levels of both VEGF and sFlt- 1 were significantly increased in HAPE patients as compared to non-HAPE individuals and recovered patients. We found that sFlt-1 was much more sensitive to hypoxia than VEGF, suggesting other pro-vascular permeability factors that also bound to sFlt-1

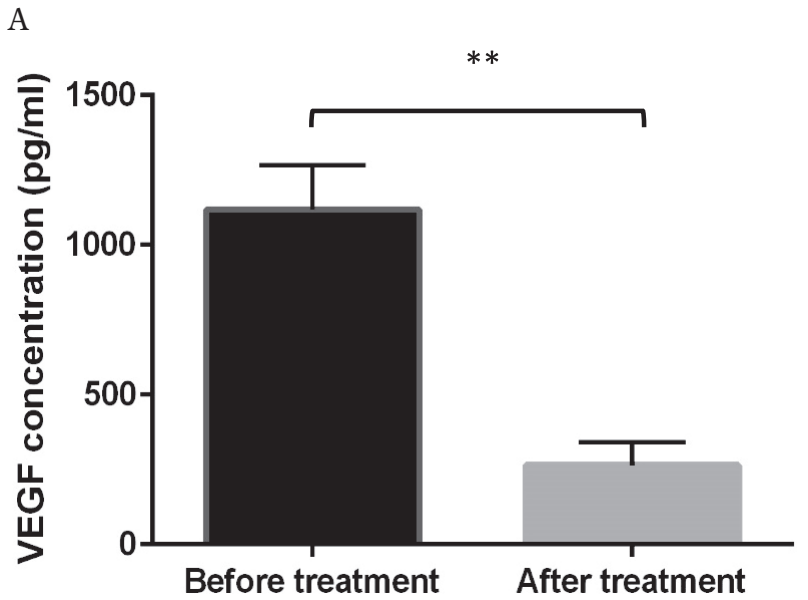

B

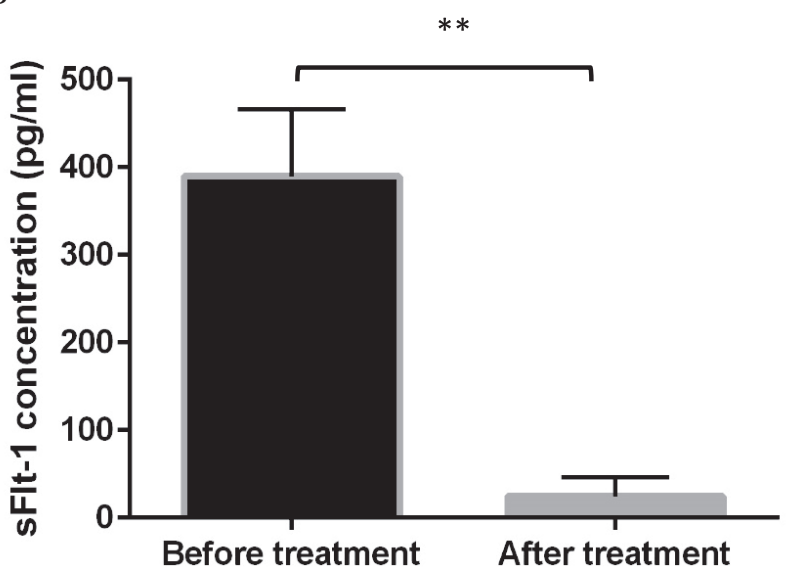

Figure 2. Plasma levels of VEGF and sFlt-1 in HAPE patients $(n=10)$ before and after treatments. A. Plasma levels of VEGF were significantly higher in the before treatment group and reduced after treatments. B. Similarly, plasma levels of sFlt-1 level were increased at the onset of HAPE and decreased after treatments. ${ }^{* *} \mathrm{p}<0.01$

might be involved as well. Our data suggested sFlt- 1 could be a better biomarker to assess HAPE. In blood samples collected from people around 5 days after entering high altitude, we observed marked increases in plasma levels of both VEGF and sFlt- 1 in HAPE patients. In contrast, a previous study showed one day after reaching high altitude, VEGF was elevated but not significantly and sFlt- 1 was even decreased. These data suggested that these two factors were not quick responding biomarkers for hypoxia and the blood collection time was crucial. In addition, in our study, after exposure to hypoxia, sFlt-1 levels were increased much more than VEGF in HAPE patients, suggesting that VEGF might not be the only factor that was involved in HAPE pathogenesis and other factors that bound with sFlt-1 and induced vascular permeability might also be involved in HAPE development $(12,13)$. To prove this concept, we compared the sFlt-1/VEGF ratios among groups to eliminate the VEGF effect and clearly demonstrated that the ratio was dramatically altered in patients before and after treatments.

Plasma levels of both VEGF and sFlt-1 were significantly increased during HAPE development and markedly reduced after treatments. In our case, sFlt-1 might contribute more to HAPE pathogenesis since it was much more sensitive to hypoxia and 

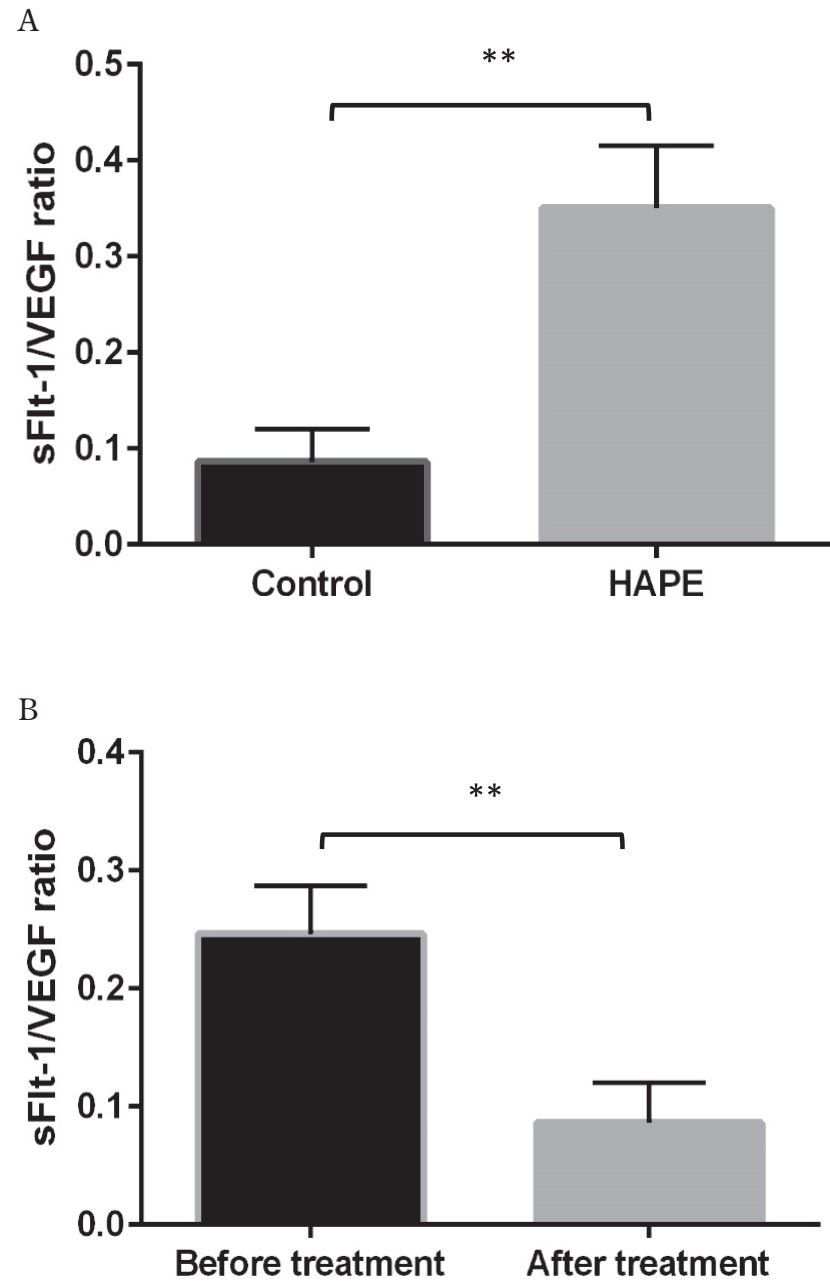

Figure 3. The difference of sFlt-1/VEGF ratios between HAPE $(n=$ 10) and control groups $(n=10)$, as well as between HAPE patients before and after treatments. A. The sFlt-1/VEGF ratio was significantly increased 4.05 folds in HAPE patients as compared to control subjects. B. The sFlt-1/VEGF ratio in the after treatment group was significantly decreased as compared with the pretreatment group. ${ }^{* *} \mathrm{p}<0.01$.

treatments as compared to VEGF response. Our data suggested sFlt-1 might be another important factor that was involved in HAPE pathogenesis and the sFlt-1/VEGF ratio could be used as a sensitive diagnostic marker for HAPE diagnosis and treatments. Although we found sFlt-1/VEGF ratio significantly decreased with the improvement of HAPE condition, other factors, such as the differences of hospital length of stay, treatments, and patients' personal constitution, might affects the results as well. In addition, despite these intriguing findings, the weakness of this study is limited sample size, future studies of larger cohorts are necessary to assess the diagnostic performance and significance of our observations linking circulating VEGF and sFlt-1 levels to HAPE condition.

\section{CONFLICT OF INTEREST}

No conflicts of interest, financial or otherwise, are declared by the author(s).

\section{ACKNOWLEDGEMENTS}

Author contributions : S.K., S.W., X.Y designed experiments ; J. L., D.J., S.M. collected data ; T.W. performed experiments ; S.M interpreted results ; X.Y. analyzed data and drafted manuscript ; Y. D. edited and revised manuscript.

\section{REFERENCES}

1. Norboo T, Saiyed HN, Angchuk PT, Tsering P, Angchuk ST, Phuntsog ST, Yahya M, Wood S, Bruce NGBall KP : Mini review of high altitude health problems in Ladakh. Journal/ Biomed Pharmacother $58: 220-225,2004$

2. Hackett PRennie D : High-altitude pulmonary edema. Journal/ JAMA 287 : 2275-2278, 2002

3. Litch JABishop RA : Reascent following resolution of high altitude pulmonary edema (HAPE). Journal/High Alt Med Biol $2: 53-55,2001$

4. Dehnert C, Berger MM, Mairbaurl HBartsch P : High altitude pulmonary edema : a pressure-induced leak. Journal/Respir Physiol Neurobiol 158 : 266-273, 2007,

5. Maloney J, Wang D, Duncan T, Voelkel NRuoss S : Plasma vascular endothelial growth factor in acute mountain sickness. Journal/Chest $118:$ 47-52, 2000

6. Dorward DA, Thompson AA, Baillie JK, MacDougall MHirani $\mathrm{N}$ : Change in plasma vascular endothelial growth factor during onset and recovery from acute mountain sickness. Journal/ Respir Med 101 : 587-594, 2007

7. Tissot van Patot MC, Leadbetter G, Keyes LE, BendrickPeart J, Beckey VE, Christians UHackett P : Greater free plasma VEGF and lower soluble VEGF receptor-1 in acute mountain sickness. Journal/J Appl Physiol (1985) 98 : 16261629,2005

8. Khalil A, Muttukrishna S, Harrington KJauniaux E : Effect of antihypertensive therapy with alpha methyldopa on levels of angiogenic factors in pregnancies with hypertensive disorders. Journal/PLoS One 3 : e2766, 2008

9. Kodama Y, Kitta Y, Nakamura T, Takano H, Umetani K, Fujioka D, Saito Y, Kawabata K, Obata JE, Mende A, Kobayashi TKugiyama K : Atorvastatin increases plasma soluble Fmslike tyrosine kinase- 1 and decreases vascular endothelial growth factor and placental growth factor in association with improvement of ventricular function in acute myocardial infarction. Journal/J Am Coll Cardiol 48 : 43-50, 2006

10. Rajakumar A, Powers RW, Hubel CA, Shibata E, von VersenHoynck F, Plymire DJeyabalan A : Novel soluble Flt-1 isoforms in plasma and cultured placental explants from normotensive pregnant and preeclamptic women. Journal/Placenta $30: 25-34,2009$

11. Maynard SE, Min JY, Merchan J, Lim KH, Li J, Mondal S, Libermann TA, Morgan JP, Sellke FW, Stillman IE, Epstein FH, Sukhatme VPKarumanchi SA : Excess placental soluble fms-like tyrosine kinase 1 (sFlt1) may contribute to endothelial dysfunction, hypertension, and proteinuria in preeclampsia. Journal/J Clin Invest 111 : 649-658, 2003

12. Acevedo LM, Barillas S, Weis SM, Gothert JRCheresh DA : Semaphorin 3A suppresses VEGF-mediated angiogenesis yet acts as a vascular permeability factor. Journal/Blood 111 : 2674-2680, 2008

13. Wu MH, Ying NW, Hong TM, Chiang WF, Lin YTChen YL: Galectin-1 induces vascular permeability through the neuropilin-1/vascular endothelial growth factor receptor-1 complex. Journal/Angiogenesis 17 : 839-849, 2014

14. Schommer K, Wiesegart N, Dehnert C, Mairbaurl HBartsch $\mathrm{P}$ : No correlation between plasma levels of vascular endothelial 
growth factor or its soluble receptor and acute mountain sickness. Journal/High Alt Med Biol $12: 323-327,2011$

15. C. Roach PB, PH, Hackett, O, Oelz, : The Lake Louise acute mountain sickness scoring system. Journal/Hypoxia and Molecular Medicine : Proceedings of the 8th International Hypoxia Symposium. 272-274, 1993

16. PH. Hackett RR : High-Altitude Medicine. 1995

17. Charnock-Jones DS, Sharkey AM, Rajput-Williams J, Burch D, Schofield JP, Fountain SA, Boocock CASmith SK : Identification and localization of alternately spliced mRNAs for vascu- lar endothelial growth factor in human uterus and estrogen regulation in endometrial carcinoma cell lines. Journal/Biol Reprod $48: 1120-1128,1993$

18. Shweiki D, Itin A, Soffer DKeshet E : Vascular endothelial growth factor induced by hypoxia may mediate hypoxiainitiated angiogenesis. Journal/Nature 359 : 843-845, 1992

19. Julian CG, Subudhi AW, Wilson MJ, Dimmen AC, Pecha TRoach RC : Acute mountain sickness, inflammation, and permeability : new insights from a blood biomarker study. Journal/J Appl Physiol (1985) 111 : 392-399, 2011 